\title{
Trasplante hepático en cirrosis por virus B: profilaxis con gammaglobulina antiHBs en dosis a "demanda"
}

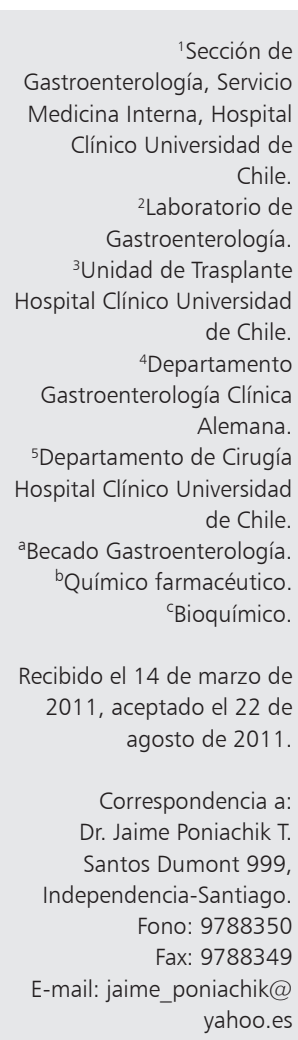

'Sección de Gastroenterología, Servicio Medicina Interna, Hospital

Clínico Universidad de Chile. ${ }^{2}$ Laboratorio de Gastroenterología.

${ }^{3}$ Unidad de Trasplante Hospital Clínico Universidad de Chile.

${ }^{4}$ Departamento Gastroenterología Clínica Alemana.

${ }^{5}$ Departamento de Cirugía Hospital Clínico Universidad de Chile.

aBecado Gastroenterología.

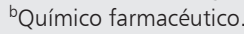
'Bioquímico.

Recibido el 14 de marzo de 2011, aceptado el 22 de agosto de 2011 .

Correspondencia a: Dr. Jaime Poniachik T. Santos Dumont 999, Independencia-Santiago. Fono: 9788350 Fax: 9788349 E-mail: jaime_poniachik@ yahoo.es

\author{
JAIME PONIACHIK ${ }^{1,3}$, CAROLINA PIZARRO ${ }^{2, a}$, JORGE CONTRERAS ${ }^{4}$, \\ JAVIER SILVA ${ }^{2}$, CARMEN HURTADO ${ }^{2, \mathrm{~b}}$, MAURICIO VENEGAS ${ }^{2, c}$, \\ JAIME CASTILLO ${ }^{3,5}$, GONZALO CARDEMIL ${ }^{3,5}$, DANNY OKSENBERG ${ }^{1,3}$, \\ JOSÉ IBARRA ${ }^{3}$, ANGÉLICA BÓRQUEZ ${ }^{3}$, JUAN CARLOS DÍAZ ${ }^{3,5}$
}

\section{Hepatitis B virus immunoglobulin on demand to prevent infection recurrence among liver allograft recipients. Report of three cases}

Infection recurrence rates among hepatitis $B$ virus infected liver allograft recipients, may be as high as $80 \%$. Immunoprophylaxis with anti HBV gammaglobulin may reduce these rates and improve survival. The dose of anti HBV gammaglobulin that must be used is not clearly defined. The most commonly accepted protocol uses 10,000 units during the anhepatic phase and 10,000 units daily during one week, followed by weekly doses of 10,000 units during one month and maintenance with 10,000 units monthly, without measuring anti hepatitis B surface antigen antibodies (antiHBs). Some reports recommend the use of immunoglobulin on demand, to maintain antiHBs titers between 100 and $250 \mathrm{U} / \mathrm{l}$. The infection recurrence rates among patients treated with immunoglobulin and Lamivudine fluctuates between 0 and $10 \%$, during follow up periods of 13 to 30 months. We report three liver allograft recipients that received immunoglobulin on demand, using a mean of 41,000 units, maintaining adequate antiHBs titers.

(Rev Med Chile 2012; 140: 78-83).

Key words: Hepatitis B virus; Immunoglobulins; Lamivudine.
$\mathrm{E}$ n la actualidad se considera que el virus de la hepatitis B (VHB) es un grave problema de salud pública y a pesar de existir una vacuna preventiva de esta enfermedad, aún sigue siendo una causa importante de daño hepático agudo, crónico y de hepatocarcinoma ${ }^{1}$.

El trasplante hepático $(\mathrm{TH})$ es un excelente tratamiento para las complicaciones severas derivadas de la infección por VHB. La infección por el VHB es responsable en alrededor de 5 a $10 \%$ de los trasplantes hepáticos reportados en la literatura ${ }^{1}$.

En el grupo de pacientes con cirrosis hepática causada por este virus, que fueron trasplantados sin un esquema de profilaxis estandarizado, hasta $80 \%$ de ellos presentó recurrencia de la infección por VHB en el órgano trasplantado ${ }^{1,2}$, ocasionando cirrosis hepática precoz, hepatitis colestásica fibrosante y hepatitis fulminante con pérdida del injerto $^{1,2}$.

Desde que se comenzó a utilizar la gammaglobulina con altos niveles de antiHBs, (HBIg) como profilaxis de la infección con el VHB, la recurrencia disminuyó entre 10 y $40 \%$ de los casos a los 5 años $^{3,4}$. De esta manera, el trasplante hepático se consolidó como una herramienta eficaz para prolongar la sobrevida. Desde ese momento la viabilidad del injerto fue similar a la reportada en el trasplante hepático indicado por otras causas ${ }^{5,6}$. Posteriormente, al incorporar a la terapia los fármacos análogos de nucleós(t)idos se ha logrado 
disminuir sostenidamente la tasa de recurrencia de la infección ${ }^{7,8}$. Reportes recientes comunican recurrencia de la infección por $\mathrm{VHB}$ en alrededor de $25 \%$ de los pacientes tratados con HBIg a 10 años, mientras que los pacientes que se trataron con HBIg asociada a análogos de nucleós(t)idos la recurrencia fue de $12 \%$ a 5 años ${ }^{9}$. El fármaco más utilizado ha sido lamivudina, sin embargo, dada su alta tasa de resistencia viral en actualidad se recomienda el uso de otros análogos de nucleós $(\mathrm{t})$ idos tales como entecavir y tenofovir ${ }^{10}$.

De un total de 125 pacientes sometidos a 135 trasplantes hepáticos entre los años 2002 y junio de 2010 en el Hospital Clínico de la Universidad de Chile, tres pacientes con cirrosis por VHB, se sometieron a trasplante hepático y fueron tratados con HBIg a dosis menores a las convencionales.

\section{Casos clínicos}

\section{Caso 1}

Paciente de 46 años, sexo femenino, con antecedentes de tuberculosis pulmonar y de hepatitis aguda a los 6 años. El año 2001 se le diagnosticó cirrosis hepática complicada con peritonitis bacteriana espontánea. El estudio etiológico demostró hepatitis crónica por $\mathrm{VHB}$, con $\mathrm{HBsAg}$, AntiHBc total, y HBeAg positivos (EIA IMx Abbott; ELFA miniVIDAS, BioMerieux). Cursó posteriormente con varias complicaciones asociadas a la hipertensión portal por lo que se la consideró candidata a trasplante hepático. En noviembre del 2003 inició tratamiento con lamivudina $150 \mathrm{mg} /$ día, logrando la negativización del $\mathrm{ADN}-\mathrm{VHB}$ por técnicas de reacción en cadena de la polimerasa (PCR).

El día 01 de abril de 2004 se le realizó el trasplante hepático, durante la fase anhepática y en el postoperatorio inmediato se le administró HBIg (Grifols ${ }^{\circledR}$ para uso IM) $5.000 \mathrm{U}$ diluidas en 300 cc de solución salina, durante 3 h y manteniendo el tratamiento antiviral con lamivudina. Las dosis posteriores de HBIg se calcularon según los títulos de anticuerpo antiHBs (EIA, IMx Abbott) Tabla 1. Se inició además, terapia inmunosupresora con metil prednisolona y ciclosporina, luego con prednisona oral y ciclosporina, asociada a quimioprofilaxis con isoniazida durante los primeros seis meses posteriores al trasplante.

La paciente evolucionó satisfactoriamente, con alta hospitalaria al duodécimo día post trasplante. Posteriormente, recibe HBIg en forma semanal durante el primer mes, y luego en régimen mensual (2.000 U IM) manteniendo lamivudina 150 mg/día. Hasta la fecha el HBsAg es indetectable.

\section{Caso 2}

Paciente de sexo masculino, 58 años, nacido en Uruguay, residente en Santiago, con antecedentes de tuberculosis pulmonar el año 1976 que se complicó con una hepatitis secundaria a isoniazida. En agosto de 2006 debuta con hepatitis aguda, cuyo estudio demostró infección aguda por VHB (HBsAg, Anti HBc total, Anti HBcIgM y HBeAg positivos) con carga viral de 3.771 .360 copias/ ml (Cobas TaqMan HBV test, Roche), además se realizó estudio de genotipo, correspondiendo al genotipo F. Evolucionó a la cronicidad con rápida progresión a la cirrosis por lo que se inició tratamiento con entecavir $0,5 \mathrm{mg} /$ día, logrando respuesta virológica al sexto mes de iniciado el tratamiento antiviral.

Durante el siguiente año, se hospitalizó en varias oportunidades por encefalopatía hepática recurrente y ascitis. En su último ingreso alcanzó puntuación MELD de 28 por lo que se consideró candidato a trasplante hepático de urgencia.

\section{Tabla 1. Títulos de anticuerpos AntiHBs y dosis de HBIg en el primer mes post trasplante hepático}

\begin{tabular}{|rcc|}
\hline Día & $\begin{array}{c}\text { Títulos AntiHBs } \\
\text { UI/L }\end{array}$ & $\begin{array}{c}\text { Dosis de } \\
\text { HBIg UI }\end{array}$ \\
\hline 1 & & 5.000 \\
2 & & 5.000 \\
\hline 3 & & 5.000 \\
\hline 4 & & 5.000 \\
\hline 5 & 5.169 & 3.000 \\
\hline 6 & 5.274 & 1.000 \\
\hline 7 & 4.894 & 1.000 \\
\hline 8 & 4.192 & 1.000 \\
\hline 9 & & 1.000 \\
\hline 11 & & 2.000 \\
\hline 14 & 2.526 & --- \\
\hline 16 & 2.074 & ---- \\
\hline 19 & 2.202 & ---- \\
\hline 23 & 1.478 & ---- \\
\hline 30 & 1.198 & --- \\
\hline & & \\
\hline
\end{tabular}


El trasplante se realizó el día 24 de marzo de 2008, evolucionando en el postoperatorio inmediato con shock hipovolémico y falla renal por necrosis tubular aguda. Recuperó rápidamente la función renal iniciando tratamiento inmunosupresor con tacrolimus (T-Inmun ${ }^{\circledR}$ ). Durante la fase anhepática y en el postoperatorio inmediato, se administró HBIg (Grifols ${ }^{\circledR}$ para uso IM) 5.000 U en 300 cc de solución salina intravenosa, manteniendo el tratamiento antiviral con entecavir. Se midieron los títulos antiHBs y HBsAg al inicio y diariamente después de cada administración de HBIg. Se observó negativización del HBsAg al octavo día posterior al trasplante. En la Tabla 2 se observan los títulos de antiHBs y la dosis administrada de HBIg.

El paciente evolucionó satisfactoriamente, con alta hospitalaria al décimo primer día post trasplante. Posteriormente, recibió HBIg en forma semanal durante el primer mes, y luego en régimen mensual (2.000 U IM) manteniendo entecavir 0,5 $\mathrm{mg} /$ día. Se utilizó quimioprofilaxis antiTBC con isoniazida durante los primeros seis meses post trasplante sin efectos adversos. Hasta la fecha el HBsAg es indetectable.

\section{Caso 3}

Paciente de 64 años, sexo masculino, con antecedentes de hipertensión arterial y diabetes mellitus tipo 2 insulino requirente. El año 2002 durante exámenes por estudio etiológico de cirrosis hepática se pesquisó HBsAg $(+)$ con detección de ADN viral por PCR (HBV fast - PharmaGen). Se complementó estudio con ecotomografía abdominal que mostró signos de daño hepático crónico e hipertensión portal, además de endoscopia digestiva alta la cual evidenció varices esofágicas pequeñas.

El 2003 se hospitalizó por encefalopatía hepática, de los exámenes destacaba la presencia de HBsAg, HBeAg y el ADN-VHB (+) por PCR, por lo cual se inició tratamiento con adefovir $10 \mathrm{mg} /$ día. $\mathrm{Al}$ décimo mes de terapia mantenía $\mathrm{HBeAg}(+)$ y $\mathrm{ADN}-\mathrm{VHB}(+)$ por lo que se decidió agregar lamivudina $150 \mathrm{mg} /$ día, logrando negativizar el ADNVHB. Durante este período presenta deterioro de la función hepática, con varios episodios de encefalopatía que requirieron de hospitalización. En agosto 2008 se observó un aumento de la carga viral hasta $10.600 \mathrm{UI} / \mathrm{ml}$. En base a esto se añade al esquema antiviral Truvada ${ }^{\circledR}$ (tenofovir $300 \mathrm{mg}$ /
Tabla 2. Títulos de anticuerpos AntiHBs y dosis de HBIg en el primer mes post trasplante hepático

\begin{tabular}{|c|c|c|}
\hline Día & $\begin{array}{c}\text { Títulos AntiHBs } \\
\text { UI/L }\end{array}$ & $\begin{array}{l}\text { Dosis de } \\
\text { HBIg UI }\end{array}$ \\
\hline 1 & & 5.000 \\
\hline 2 & 58 & 5.000 \\
\hline 3 & 129 & 8.000 \\
\hline 4 & 423 & 8.000 \\
\hline 5 & 1.022 & 5.000 \\
\hline 6 & & 4.000 \\
\hline 7 & & 4.000 \\
\hline 8 & 1.410 & 2.000 \\
\hline 10 & 1.430 & ---- \\
\hline 11 & 830 & ---- \\
\hline 12 & 670 & 3.000 \\
\hline 13 & 680 & ---- \\
\hline 18 & 1.130 & ---- \\
\hline 20 & 890 & ---- \\
\hline 21 & & 2.000 \\
\hline 24 & 920 & ---- \\
\hline 27 & 12 & ---- \\
\hline 30 & 1.048 & ---- \\
\hline
\end{tabular}

emtricitabina $200 \mathrm{mg}$ ) logrando descenso de carga viral a $623 \mathrm{UI} / \mathrm{ml}$. En dos estudios con imágenes (TC y RM) se observan al menos tres lesiones focales hepáticas hipercaptantes compatibles con hepatocarcinoma.

Se realizó trasplante hepático ortotópico el 26 de julio 2009, administrándole durante la fase anhepática y en el postoperatorio inmediato HBIg (Grifols ${ }^{\circledR}$ para uso IM) $3.000 \mathrm{U}$ en $300 \mathrm{cc}$ de solución salina intravenosa, durante 3 h y manteniendo el tratamiento antiviral con adefovir, lamivudina y Truvada ${ }^{\circledR}$. Las dosis posteriores de HBIg se calcularon según los títulos de anticuerpo antiHBs (EIA, IMx Abbott) Tabla 3. Se inició además, terapia inmunosupresora con metil prednisolona asociada a tacrolimus y quimioprofilaxis antiTBC con isoniazida durante los primeros seis meses posteriores al trasplante.

Evolución inicial satisfactoria, sin embargo, cursó posteriormente con sepsis por Staphylococcus aureus e infección por citomegalovirus. Se dio 
Profilaxis con HBlg en trasplante hepático por VHB - J. Poniachik et al

Tabla 3. Títulos de anticuerpos AntiHBs y dosis de HBIg en el primer mes post trasplante hepático

\begin{tabular}{|c|c|c|}
\hline Día & $\begin{array}{c}\text { Títulos AntiHBs } \\
\text { UI/L }\end{array}$ & $\begin{array}{c}\text { Dosis de } \\
\text { HBIg UI }\end{array}$ \\
\hline 1 & 41 & 3.000 \\
\hline 2 & 38 & 3.000 \\
\hline 3 & 54 & 3.000 \\
\hline 4 & & 2.000 \\
\hline 5 & & 2.000 \\
\hline 6 & 45 & 2.000 \\
\hline 7 & 35 & 1.000 \\
\hline 8 & 25 & 3.000 \\
\hline 10 & 23 & 5.000 \\
\hline 11 & 74 & 5.000 \\
\hline 12 & & 5.000 \\
\hline 13 & & 5.000 \\
\hline 14 & 452 & 2.000 \\
\hline 15 & 392 & 3.000 \\
\hline 17 & 579 & ---- \\
\hline 18 & & 1.000 \\
\hline 19 & & 1.000 \\
\hline 21 & 371 & ---- \\
\hline 25 & 420 & 2.000 \\
\hline 30 & & 2.000 \\
\hline
\end{tabular}

de alta con indicación de administración mensual de HBIg (2.000 U IM) manteniendo tratamiento antiviral con Truvada ${ }^{\circledR}$ un comprimido/día. Hasta la fecha el HBsAg es indetectable.

\section{Discusión}

En este estudio se han presentado tres casos de trasplante hepático en pacientes con cirrosis por VHB, manejándose en forma exitosa con dosis reducida y a "demanda" de HBIg.

Con la utilización de inmunoprofilaxis mediante $\mathrm{HBIg}$, los resultados del trasplante hepático han mejorado significativamente. Estos anticuerpos de donantes antiHBs son policlonales y van dirigidos contra la envoltura del virus, causando la neutralización viral y por ende la prevención de la infección de nuevos hepatocitos. Con este método se ha comunicado una efectividad aproximada de $80 \%{ }^{3,4,9}$.

No existe consenso en la dosis recomendada de HBIg. El protocolo más aceptado utiliza el uso de 10.000 UI endovenosa durante la fase anhepática del trasplante, seguido de 10.000 UI diarias por una semana, para continuar con 10.000 UI semanales, durante el primer mes y luego 10.000 UI mensuales en forma indefinida ${ }^{11}$.

En otros estudios se han utilizado dosis entre 10.000 y 5.000 UI endovenosa por día durante la primera semana, seguidas de 5.000 UI endovenosa tres veces a la semana en la segunda semana y luego 5.000 UI mensuales en forma indefinida ${ }^{12,13}$. También se ha reportado en una serie pequeña el uso de HBIg intramuscular desde la fase anhepática del trasplante (2.000 UI en ese momento, seguido de la administración de 2.000 UI diarias por siete días y posteriormente 2.000 UI mensuales en forma indefinida) logrando mantener HBsAg negativo al año de seguimiento ${ }^{14}$.Otros estudios más recientes utilizan entre 400 a 800 UI vía IM en forma mensual asociado a Lamivudina con resultados bastante promisorios ${ }^{15}$.

La recurrencia de la infección por el VHB con el uso de HBIg en dosis altas, como terapia única, varía entre 7 y $23 \%$ con seguimientos entre 2 y 55 meses. Sin embargo, el costo de esta profilaxis es elevado, siendo un gran problema de salud pública sobre todo en Latinoamérica, donde las políticas del sistema público son limitadas. Para reducirlo, algunos estudios clínicos sugieren la utilización de dosis bajas de HBIg indicada "a demanda", guiada según títulos de antiHBs, en forma única o asociada al uso de análogos de nucleós(t)idos. De esta manera se logra ahorrar recursos manteniendo la efectividad de la profilaxis en la prevención de la recidiva de la infección por VHB en el posttrasplante hepático ${ }^{12}$.

En nuestros pacientes utilizamos en promedio 41.000 UI durante el primer mes, lo que significa una reducción de $63 \%$ de la dosis estándar de HBIg. Esto nos permitió reducir el costo en aproximadamente 31.000 dólares por cada paciente.

Aún no está claro cuál es el nivel seguro de antiHBs, pero se recomiendan títulos en el rango comprendido entre 100 y 250 UI/l según el período post THO. Probablemente en los pacientes de alto riesgo sea recomendable mantener títulos entre 250 y $500 \mathrm{UI} / \mathrm{l}$. Este último grupo de pacientes 
corresponde a aquellos que no lograron reducir la carga viral previo al trasplante. En el paciente $\mathrm{n}^{\circ} 1$ se documentaron títulos sobre 100 al quinto día post trasplante, en el paciente $\mathrm{n}^{\circ} 2$ al tercer día, mientras que en el paciente $n^{\circ} 3$ se consiguieron en el día 14 post trasplante, todos negativizando el HBsAg. Probablemente algunos pacientes necesiten títulos de antiHBsAg menores a 100 UI/l como ocurrió en el caso $n^{\circ} 3$.

Por la existencia de reservorios extrahepáticos del VHB, la reinfección del injerto es de alta frecuencia, más aun en el grupo de alto riesgo (carga viral alta al momento del trasplante). La reinfección del injerto puede tener un pronóstico generalmente ominoso de no ser tratado, ocasionando cirrosis, hepatitis colestásica fibrosante y hepatitis fulminante con pérdida del injerto en un corto período ${ }^{1,2}$.

Lo ideal es que los pacientes lleguen al trasplante con baja replicación viral, para lo cual se recomienda previo al trasplante mantener terapia con análogos de núcleos(t)idos. En el primer caso mencionado se indicó lamivudina, en el segundo entecavir y en el tercer paciente la combinación de lamivudina, adefovir, tenofovir y emtricitabina. En los pacientes $\mathrm{n}^{\circ} 1 \mathrm{y} \mathrm{n}^{\circ} 2$ no se detectó $\mathrm{ADN}$ viral por PCR previo al trasplante, mientras que el paciente 3 redujo significativamente la carga viral previo a la intervención (menor a $1 \times 10^{3} \mathrm{UI} / \mathrm{ml}$ ). En el estudio de Samuel et al, se reportó recurrencia de la infección por VHB a dos años del trasplante en $96 \%$ de los pacientes que tenían PCR positiva previo al trasplante vs $29 \%$ de los pacientes en los que no se detectó ADN viral por PCR antes de la intervención ${ }^{3}$.

En los tres casos presentados se ha utilizado esquemas de terapia con HBIg en dosis bajas y ajustadas según títulos de anticuerpos antiHBs. Al momento del trasplante se utilizó en todos los pacientes, gammaglobulina de uso intramuscular (Grifol ${ }^{\circledR}$ ) pero administrada en forma endovenosa, diluida en $300 \mathrm{cc}$ de solución salina, administrada en forma lenta $(3 \mathrm{~h})$ para evitar reacciones adversas. No se reportó ningún efecto adverso por este motivo en los casos presentados.

En relación a la duración del tratamiento, se sugiere que éste debe ser indefinido dado que, a pesar de que los pacientes presenten HBsAg negativos, es posible detectar aún $\mathrm{ADN}$ viral en el suero, linfocitos y hepatocitos entre 50 y $70 \%$ de los $\operatorname{casos}^{1,3}$.
Alternativamente se ha sugerido la inmunización activa mediante la administración de vacunas recombinantes, que incluyan los antígenos S, preS1 y preS2 y aquellas con inmunoestimulantes adyuvantes ${ }^{10}$. Esto último, se ha ensayado en enfermos sin evidencia de replicación viral previa y que han recibido HBIg a lo menos por dieciocho meses después del trasplante, sin evidencia de recurrencia. En un estudio español se usaron dos ciclos de vacunación, de tres dosis de $40 \mu \mathrm{g}$ (tiempo cero, al mes y sexto mes), logrando títulos de antiHBs sobre $100 \mathrm{UI} / \mathrm{L}$ en $24 \%$ de los casos $^{16}$. Algunos reportes recientes sugieren que en los pacientes HBsAg positivos la vacunación debería efectuarse previo al trasplante ${ }^{10}$. Estos resultados deben ser corroborados en un número mayor de enfermos y en estudios multicéntricos controlados.

En estos tres casos reportamos el uso exitoso de gammaglobulina en dosis reducida a "demanda", observándose que puede ser una práctica costoefectiva, especialmente en países como el nuestro, donde las políticas de salud pública deben priorizar problemas de salud que sean de alto impacto $\mathrm{y}$ a costos razonables.

\section{Referencias}

1. O'Grady JG, Smith HM, Davies SE, Daniels HM, Donaldson PT, Tan KC, et al. Hepatitis B virus reinfection after orthotopic liver transplantation. Serological and clinical implications. J Hepatol 1992; 14 (1): 104-11.

2. Davies SE, Portmann BC, O'Grady JG, Aldis PM, Chaggar K, Alexander GJ, et al. Hepatic histologic findings after transplantation for chronic hepatitis B virus infection, including a unique pattern of fibrosing cholestatic hepatitis. Hepatology 1991; 13 (1): 150-7.

3. Samuel D, Bismuth A, Mathieu D, Arulnaden JL, Reynes M, Benhamou JP, et al. Passive immunoprophylaxis after transplantation in HBsAg-positive patients. Lancet 1991; 337 (8745): 813-5.

4. Gugenheim J, Crafa F, Fabiani P, Goubaux B, Ouzan D, Mouiel J. Long-term passive immunoprophylaxis of $B$ virus recurrence after liver transplantation in HBs antigen-positive patients. Transp Proc 1993; 25 (1 Pt 2): 1349-50.

5. Terrault N, Wright TL. Hepatitis B virus infection and liver transplantation. Gut 1997; 40 (5): 568-71.

6. Samuel D, Muller R, Alexander G, Fassati L, Ducot B, Benhamou JP, et al. Liver transplantation in european patients with hepatitis B surface antigen. N Engl J Med 1993; 329 (25): 1842-7. 
7. Markowitz JS, Martin P, Conrad AJ, Markmann JF, Seu P, Yersiz H, et al. Prophylaxis against hepatitis B recurrence following liver transplantation using combination lamivudine and hepatitis B immune globulin. Hepatology 1998; 28 (2): 585-9.

8. Han SH, Ofman J, Holt C, King K, Kunder G, Chen P, et al. An efficacy and cost-effectiveness analysis of combination hepatitis B immune globulin and lamivudine to prevent recurrent hepatitis B after orthotopic liver transplantation compared with hepatitis B immune globulin monotherapy. Liver Transpl 2000; 6 (6): 741-8.

9. Roche B, Feray C, Gigou M, Roque-Afonso AM, Arulnaden JL, Delvart V, et al. HBV DNA persistence 10 years after liver transplantation despite successful anti-HBS passive immunoprophylaxis. Hepatology 2003; 38 (1): 86-95.

10. Beckebaum S, Sotiropoulos GC, Gerken G, Cicinnati VR. Hepatitis B and liver transplantation: 2008 update. Rev Med Virol 2009; 19 (1): 7-29.

11. Lok AS. Prevention of recurrent hepatitis B post-liver transplantation. Liver Transpl 2002; 8(10 Suppl 1): S6773.

12. Di Paolo D, Tisone G, Piccolo P, Lenci I, Zazza S, Angelico M. Low-dose hepatitis B immunoglobulin given "on demand" in combination with lamivudine: a highly cost-effective approach to prevent recurrent hepatitis B virus infection in the long-term follow-up after liver transplantation. Transplantation 2004; 77 (8): 1203-8.

13. Terrault NA, Zhou S, Combs C, Hahn JA, Lake JR, Roberts JP, et al. Prophylaxis in liver transplant recipients using a fixed dosing schedule of hepatitis B immunoglobulin. Hepatology 1996; 24 (6): 1327-33.

14. Alonso I, Herreros de Tejada A, Moreno JM, Rubio E, Lucena JL, De la Revilla J, et al. Effectiveness of lowdose intramuscular anti-VHB immune globulin in the prophylaxis of viral B hepatitis reinfection after liver transplantation: preliminary report. Transplant Proc 2003; 35 (5): 1850-1.

15. Gane EJ, Angus PW, Strasser S, Crawford DH, Ring J, Jeffrey GP, et al. Lamivudine plus low-dose hepatitis B immunoglobulin to prevent recurrent hepatitis B following liver transplantation. Gastroenterology 2007; 132 (3): 931-7.

16. Sánchez-Fueyo A, Rimola A, Grande L, Costa J, Más A, Navasa $M$, et al. Hepatitis B immunoglobulin discontinuation followed by hepatitis B virus-vaccination: a new strategy in the prophylaxis of hepatitis B virus recurrence after liver transplantation. Hepatology 2000; 31 (2): 496-501. 